\title{
The Inefficiency of the Stock Market Equilibrium
}

\author{
JOSEPH E. STIGLITZ \\ Princeton University
}

\begin{abstract}
This paper establishes that when there is not a complete set of markets but more than one commodity the stock market equilibrium will not in general be a constrained Pareto optimum. The economy will lack both the property of exchange and production efficiency. Necessary conditions which must be satisfied if the economy is to be a constrained Pareto optimum for all technologies are derived; if all individuals have identical, homothetic indifference maps, then either there must be unitary price elasticities (so there is no effective risk) or all individuals must have the same degree of risk aversion (so there is no trade on the stock market).
\end{abstract}

This paper is one of a series attempting to explore the efficiency with which the economy allocates resources in the presence of uncertainty. Arrow's classic paper (1964) established a set of sufficient conditions under which the market equilibrium was a Pareto optimum. He required that there be a complete set of risk markets, a condition which was obviously not satisfied in any economy. Karl Borch (1962) showed that when there was not a complete set of markets, the market equilibrium would not be a Pareto optimum. These results engendered a search for a weaker set of conditions under which the market equilibrium might satisfy a perhaps weaker sense of optimality.

Ideally, one would like to have a theory which explains incompleteness of markets; several explanations have been offered associated with transactions costs and imperfections in information. An evaluation of the market economy should then entail a comparison with a socialist economy facing the same transactions technology or the same information problems. In such a comparison, the market economy might be found deficient with respect to the set of markets which operate. The discussion of this paper can be viewed as a "minimal" test of the market economy: given the set of markets operating does it allocate resources efficiently; if it does, it still does not mean that one should view the market economy as being efficient, but if it does not, it clearly can be viewed as being inefficient.

This still leaves us with the problem of defining some notion of "constrained efficiency". One way of posing the problem is to ask, if we replaced the current managers of enterprises, who make their production decisions so as to maximize the market value of the firm, with "public managers" who base their production decisions on some other criterion, is it possible that with the new resource allocation everyone could be better off, assuming that the profits of the firm are still distributed to shareholders in proportion to their ownership shares (and that no new risk markets are opened up). In addition, we can ask, is it possible, by assigning ownership shares in the various firms in the economy (rather than allowing these to be determined by free exchange) to make everyone better off? Finally, we can enquire whether there are "simple" interventions in the market economy-e.g. a set of taxes and subsidies-which would make some group better off without making anyone worse off? This, however, may still provide what some might regard as an unfair test of the market economy: if the taxes-subsidies are state 
contingent, the government may have effectively introduced an additional security (or set of securities) into the economy. To avoid this criticism, we wish to tie the government's hands even further: we require any taxes and subsidies to be not state dependent. We need to emphasize that what we are proposing is a minimal test of the efficiency of the market economy; even if it passes this test, there may be grounds for government intervention, either in taking actions which affect the set of markets which operate or in introducing state-contingent taxes and subsidies.

In the case of a simple stock market economy with a single commodity the test which we are proposing is equivalent to the notion of constrained Pareto optimality introduced by Diamond (1967). In an economy with a stock market, each individual purchases a fraction of each of the firms. In addition, the individual may borrow (or lend) at the safe rate of interest. Diamond considered an economy in which these were the only securities allowed, and contrasted the market allocation where firms maximized their stock market value, with that where the government allocated all resources, but was severely restricted in its ability to distribute the output of the economy: each individual received a linear function of the output of the different firms. He then showed that, (a) if the technology of each firm exhibited stochastic homotheticity (so that the ratio of the output in any two states was independent of scale); and (b) each firm believed that its market value was proportional to its scale, then the market equilibrium would be a constrained Pareto optimum.

The objective of this paper is to show that, even under the extremely favourable conditions for the market economy which Diamond hypothesized, the stock market allocation of resources is not a constrained Pareto optimum when there are two or more outputs. It should be noted that the model we formulate is consistent with the standard "spanning" assumptions and the factor models often employed in the finance literature. Thus, our analysis shows that the widespread view that in these models the market equilibrium is efficient is only true, in general, for the special case where there is a single commodity.

There are a number of intuitive explanations of this result. Any change in the allocation of investment or the distribution of ownership shares in the different assets will, in general, change the distribution of prices. Hence the nature of the risky assets which are marketed changes. Since there are incomplete risks markets, this change in the nature of the risky assets which are available has welfare effects which need to be taken into account. Competitive firms, which are price takers, will ignore the effects of their actions on the price distribution. There are two sets of restrictions on preferences which will guarantee that the market will be efficient regardless of the probability distribution of output.

These restrictions ensure either that the individual does not care about the price distribution or that the distribution of ownership claims or investment allocations leave the price distribution unaltered. The distribution of ownership claims has no effect on price distributions only if all individuals have identical, homothetic indifference curves but changes in the allocation of investment have no effects only when there is no trade (because all individuals are identical) or when there is no effective risk (the value of output is constant, because all individuals have the same demand curves with unitary price elasticity).

The argument may be put another way. With a complete set of risks markets, we know we wish to equalize the marginal rates of substitution between any two states for all individuals. With an incomplete set of markets, we cannot do this, but we may be able to have a more "efficient" distribution of risks (come closer to equalizing, on average, the marginal rates of substitution) if we can change the price distribution (and thus the "profit distribution") associated with the risky asset. The government recognizes that it can change this price distribution by altering the allocation of investment and the ownership shares in the different assets. The market ignores this effect. 
The matter may be put in still another way. With a single output there is no distinction between stochastic homotheticity for a firm or for an industry: the ratio of output or profits in any two states of nature remains a constant. With more than one commodity, if a single firm increases its scale, it may perceive that the ratio of its profits in any two states of nature remains constant; but when the industry expands its scale, the price of the output of the industry may change differently in different states, so that the ratio of industry profits in two different states changes as the scale of the industry changes; there is private stochastic homotheticity-taking prices in each state of nature as given-but not social stochastic homotheticity. The former assures that firms will wish to maximize stock market value $\mathrm{B}^{2.3}$ and makes it reasonable for them to assume that value is proportional to their scale. But the latter is what is necessary to ensure constrained optimality of the market solution.

At a more formal level, the difference between the competitive equilibrium and the constrained optimum arises from the fact that prices enter into the constraints on the distribution of profits (i.e. each individual receives a given fraction of the profits of the firm, regardless of the state of nature); when the socialist planner changes an allocation of ownership claims and patterns of investment, it takes into account how these decisions affect prices; in a competitive economy, this "price distribution effect" is always ignored by firms. Even extending the set of markets-introducing futures markets-may not lead to (constrained) optimality. ${ }^{4}$

Although Stiglitz (1975a) and Hart (1975) had earlier noted the non-optimality of the stock market allocation when there was more than one output, they provided a less complete characterization of the nature of the inefficiency than we are able to provide here. $^{5}$

We shall attempt to provide a set of necessary and sufficient conditions for the market equilibrium to be a constrained Pareto optimum. We have not yet succeeded in providing a complete characterization, but our basic results show that there is an extremely strong presumption that the market equilibrium will not be a constrained Pareto optimum. Only if the structure of the economy is such that risk itself does not matter can we be sure that the economy is efficient.

Section 2 presents the basic model and two sufficient conditions for the optimality of the stock market equilibrium. Sections 3 and 4 present necessary conditions for the optimality of the stock market equilibrium. Section 5 relates our results to other results on the optimality of the stock market equilibrium.

\section{THE MODEL}

\subsection{Market equilibrium}

The simplest model for establishing the non-optimality of the stock market allocation is one involving two commodities, denoted by subscripts 1 and 2 . W'e let the first commodity be our numeraire, and denote the relative price of the two commodities by $p$. The $j$-th individual's utility is a function of the relative price, $p$, and his income $Y^{j}$ :

$$
v^{i}=V^{l}\left(p, Y^{i}\right)
$$

( $V$ is the indirect utility function). The individual's income (wealth) next period depends on how he allocates his portfolio. For simplicity, we assume all technological risk resides in the second industry, and that it has multiplicative uncertainty and constant returns to scale, i.e.

$$
X_{2}=g(\theta) I_{2}
$$

where $X_{2}$ is the output of the second industry in state $\theta$, when the investment level is $I_{2}$. (The generalization to the case of multiplicative uncertainty with diminishing returns to scale is straightforward.) We can let $g(\theta) \equiv \theta$. (This simplifies the notation.) The first 
industry is assumed to be perfectly safe:

$$
X_{1}=(1+r) I_{1} \text {. }
$$

Thus, if the $j$-th individual has an initial wealth of $I^{j}$, and he invests a fraction, $\alpha^{j}$, in the first industry and the remainder in the second

$$
Y^{i}=\left[\alpha^{i}(1+r)+\left(1-\alpha^{i}\right) p \theta\right] I^{i}
$$

In our model, it makes no difference whether we think of the individual as investing in the production processes directly or investing in a firm which invests in the production process. This follows from the assumptions of technology (constant returns to scale) and competition. In market equilibrium, the value of any firm in the $i$-th industry must be equal to its investment. Under the competitive hypothesis, it will believe that by increasing investment it will increase its market value proportionately; the net worth of the original shareholders is thus increased so long as the value of the firm $V_{i}$, exceeds $I_{i}$. Thus, investment continues until $V_{i}=I_{i}$. Since all shareholders believe that the action of the firm will have no effect on prices in any state of nature, shareholders will only be concerned with net worth, and thus will wish the firm to maximize their market value (cf. footnote 2 above).

Thus, let $\beta^{j}$ be the $j$-th individual's ownership share in industry 2 and $\gamma^{\prime}$ be his share in industry 1. (Again, because of our assumptions, all firms within an industry are identical and have perfectly correlated returns, so we need not distinguish among them.) Then

$$
\begin{aligned}
\left(1-\alpha^{\prime}\right) I^{\prime} & \equiv \beta^{\prime} I_{2}, \quad \sum \beta^{\prime}=1 \\
\alpha^{\prime} I^{\prime} & \equiv \gamma^{\prime} I_{1}, \quad \sum \gamma^{\prime}=1 \\
I_{2} & =\sum\left(1-\alpha^{\prime}\right) I^{j} \\
I_{1} & =\sum \alpha^{\prime} I^{\prime} .
\end{aligned}
$$

The $j$-th individual takes the relationship between $p$ and $\theta$ as given, independent of his actions; thus the optimal portfolio allocation for the $j$-th individual is given by

$$
\max _{\alpha^{\prime}} E V^{\prime}\left(p,\left(\alpha^{j}(1+r)+\left(1-\alpha^{\prime}\right) p \theta\right) I^{\prime}\right)
$$

i.e.

$$
E V_{Y}^{j}((1+r)-p \theta)=0
$$

which implies that

$$
1+r=\frac{E V_{Y}^{\prime} p \theta}{E V_{Y}^{\prime}}=\frac{E V_{Y}^{k} p \theta}{E V_{Y}^{k}}, \quad \text { all } j, k
$$

the weighted expected returns from the risky asset must be equal to the safe return, where the weights are relative marginal utilities.

Market equilibrium with rational expectations in this simple model is characterized by (9) and just one more equation: the relationship between $p$ and $\theta$ which individual's expect is the actual market relationship. In the market equilibrium, we require that the (consumption) demand for commodity 2 (say) be equal to its supply, i.e. aggregate supply is given by

$$
X_{2}\left(\theta, I_{2}\right)=\theta I_{2} \text {. }
$$

The demand by the $j$-th individual is just a function of the price and his income:

$$
C_{2}^{i}=C_{2}^{j}\left(p, Y^{i}\right)
$$

(where $Y^{j}$ is given by (4)). (The demand curve may be derived easily from the indirect 
utility function by Roy's formula:

$$
C_{2}^{j}=-V_{p}^{j} / V_{Y}^{j}
$$

we shall make use of this result later.)

Aggregate demand is thus given by

$$
C_{2} \equiv \sum_{i} C_{2}^{i} \text {. }
$$

Thus $p(\quad)$ is given by the solution to

$$
C_{2}=\theta I_{2}
$$

\subsection{Constrained Pareto optimum}

This market solution now needs to be contrasted with a constrained Pareto optimum. We assume (as did Diamond) that the government can control (a) the allocation of shares of profits and (b) the levels of production of firms. This is done to maximize, say, $E V^{1}$, given the expected utility levels of all other individuals. Thus the government maximizes, for some set of $\lambda^{j}$,

$$
\underset{\left\{\hat{\beta}^{\prime}, \hat{\gamma}^{i}, f_{2}\right\}}{E} \lambda^{j} V^{j}\left(p, \dot{Y}^{j}\right)
$$

where now

$$
\hat{Y}^{i}=\hat{\beta}^{i} \theta p \hat{I}_{2}+\hat{\gamma}^{i}(1+r)\left(\vec{I}-\hat{I}_{2}\right)
$$

where $\hat{\beta}^{i}$ is the fraction of the output of firm 2 assigned to the $j$-th individual, $\sum \hat{\beta}^{\prime}=1$ and similarly, $\hat{\gamma}^{j}$ is the fraction of the output of firm 1 assigned to the $j$-th individual, $\sum \hat{\gamma}^{i}=1$, and $\bar{I}$ is the total level of investment, $\bar{I}=\sum I^{\prime}$.

This yields the first order conditions

$$
\begin{array}{r}
\lambda^{j} E V_{Y}^{j}(1+r)-\lambda^{1} E V_{Y}^{1}(1+r)+\frac{1}{I_{1}} E B \frac{d p(\theta)}{d \gamma^{\prime}}=0 \\
\lambda^{j} E V_{Y}^{\prime} p \theta-\lambda^{\prime} E V_{Y}^{1} p \theta+\frac{1}{I_{2}} E B \frac{d p(\theta)}{d \beta^{\prime}}=0 \\
E \sum \lambda^{i} V_{Y}^{i}\left(\hat{\beta}^{\prime} \theta p-\hat{\gamma}^{i}(1+r)\right)+E B \frac{d p(\theta)}{d I_{2}}=0
\end{array}
$$

where

$$
\begin{aligned}
B & =\sum \lambda^{i}\left(V_{p}^{i}+V_{Y}^{\prime} \hat{\beta}^{\prime} I_{2} \theta\right) \\
& =\sum \lambda^{i} V_{Y}^{\prime}\left(\hat{\beta}^{\prime} \theta I_{2}-C_{2}^{\prime}\right)
\end{aligned}
$$

(using Roy's identity), and where, using (14)

$$
\begin{gathered}
\frac{1}{I_{1}} \frac{d p(\theta)}{d \gamma^{i}}=-\frac{\left(\frac{\partial C_{2}^{1}}{\partial Y}-\frac{\partial C_{2}^{1}}{\partial Y}\right)(1+r)}{\sum_{k}\left(\frac{\partial C_{2}^{k}}{\partial p}+\frac{\partial C_{2}^{k}}{\partial Y} \hat{\beta}^{k} \theta I_{2}\right)} \\
\frac{1}{I_{2}} \frac{d p(\theta)}{d \beta^{i}}=-\frac{\left(\frac{\partial C_{2}^{j}}{\partial Y}-\frac{\partial C_{2}^{1}}{\partial Y}\right) p \theta}{\sum_{k}\left(\frac{\partial C_{2}^{k}}{\partial p}+\frac{\partial C_{2}^{k}}{\partial Y} \hat{\beta}^{k} \theta I_{2}\right)}
\end{gathered}
$$


and

$$
\frac{d p(\theta)}{d I_{2}}=-\frac{\sum_{k} \frac{\partial C_{2}^{k}}{\partial Y}\left(\hat{\beta}^{k} p \theta-\hat{\gamma}^{k}(1+r)\right)}{\sum_{k}\left(\frac{\partial C_{2}^{k}}{\partial p}+\frac{\partial C_{2}^{k}}{\partial Y} \hat{\beta}^{k} \theta I_{2}\right)} .
$$

What is important to observe in (16) is that the effect of the allocation of investment, the distribution of income and the allocation of ownership claims on the risky firm on the price distribution all need to be taken into account. Since in competitive markets, the effects on price distribution are (by assumption) ignored, competitive market allocations will not, in general, be (constrained) Pareto optima.

(a) Exchange Efficiency. The conditions (16a) and (16b) can be thought of as the conditions for exchange efficiency, given the allocation of investment. Dividing (16b) by (16a), we obtain (using (18a) and (18b))

$$
\frac{E V_{Y}^{1} p \theta}{E V_{Y}^{1}(1+r)}=\frac{\lambda^{j} E V_{Y}^{j} p \theta+E A^{\prime} B p \theta}{\lambda^{\prime} E V_{Y}^{j}(1+r)+E A^{\prime} B(1+r)}
$$

where

$$
A^{i}=\frac{-\left(\frac{\partial C_{2}^{\prime}}{\partial Y}-\frac{\partial C_{2}^{1}}{\partial Y}\right)}{\sum_{k} \frac{\partial C_{2}^{k}}{\partial p}+\frac{\partial C_{2}^{k}}{\partial Y} \hat{\beta}^{k} \theta I_{2}} .
$$

Contrasting (9) and (19), we see that a necessary condition for the exchange efficiency of the market is that

$$
\frac{E A^{\prime} B p \theta}{E V_{Y}^{\prime} p \theta}=\frac{E A^{i} B}{E V_{Y}^{\prime}}
$$

It is apparent that (20) will not, in general, be satisfied. Sufficient conditions for exchange efficiency are: ${ }^{6}$

(i) $A^{j} \equiv 0$, i.e. the marginal propensity to consume good 2 is the same for all individuals.

$$
\frac{\partial C_{2}^{\prime}}{\partial Y}=\frac{\partial C_{2}^{1}}{\partial Y}, \text { all } j
$$

This must hold at all prices and incomes. The implications of this for indifference maps have been extensively studied elsewhere. If all individuals have identical, homothetic indifference maps, clearly (21) is satisfied.

This condition assures us that the distribution of income has no effect on prices (and hence on the price distribution).

(ii) $B=0$. To interpret this condition, we define

$$
\begin{aligned}
T^{i} & =\hat{\beta}^{i} \theta I_{2}-C_{2}^{i} \\
& =\left(1-\alpha^{i}\right) I^{i} \theta-C_{2}^{i} .
\end{aligned}
$$

$\beta^{\prime} \theta I_{2}$ is the individual's "ownership" of $X_{2}$ (through his share in the firms in this industry). Thus $T^{j}$ is the (implicit) net trade of the $j$-th individual. ( $T^{\prime}>0$ implies that the individual is selling $X_{2}$ net, $T^{\prime}<0$ implies that he is buying $X_{2}$.) 
Substituting (22) into (17), we obtain

$$
B \equiv \sum \lambda^{i} V_{Y}^{i} T^{\prime} .
$$

$V_{Y}^{\prime} T^{j}$ measures the effect of a change in price on the $j$-th individual's welfare. If $T^{i}=0$, the individual is, in effect, neither buying nor selling the risky commodity, so changes in the price neither make him better nor worse off. The condition $B=0$ says that the weighted average value of the welfare changes from a change in price (where the weights are the social marginal utilities of income) must be zero, for all (relevant) values of $p$. This is an extremely restrictive assumption, as our subsequent analysis will make clear. For now, we note two sufficient conditions for $B=0$ and hence for the exchange efficiency of the economy are

(i) all individuals are identical, so $T^{i}=0$, or

(ii) all individuals have Cobb-Douglas utility functions with possibly different exponents; in this case $p \theta$ is constant; there is no risk and hence the economy is a constrained Pareto optimum.

(b) Production efficiency. From (16a), we obtain

$$
\hat{\gamma}^{\prime} \lambda^{i} E V_{Y}^{\prime}=\hat{\gamma}^{i} \lambda^{i} E V_{Y}^{1}-\hat{\gamma}^{\prime} E B A^{\prime} \text {. }
$$

Summing over all $j$,

Similarly,

$$
\begin{aligned}
\sum_{j} \hat{\gamma}^{j} \lambda^{j} E V_{Y}^{\prime} & =\sum_{j} \hat{\gamma}^{\prime} \lambda^{1} E V_{Y}^{1}-\sum_{j} E B A^{\prime} \hat{\gamma}^{\prime} \\
& =\lambda^{1} E V_{Y}^{1}-\sum_{i} E B A^{\prime} \hat{\gamma}^{\prime} .
\end{aligned}
$$

$$
\sum_{j} \hat{\beta}^{\prime} \lambda^{\prime} E V_{Y}^{i} p \theta=\lambda^{1} E V_{Y}^{1} p \theta-E B \sum_{,} A^{\prime} \hat{\beta}^{\prime} p \theta .
$$

Substituting into $(16 \mathrm{c})$, we obtain

$$
\lambda^{1} E V_{Y}^{1}(p \theta-(1+r))+E B \frac{d p}{d I_{2}}-E B\left(\sum A^{i}\left(\hat{\beta}^{\prime} p \theta-\hat{\gamma}_{1}(1+r)\right)=0 .\right.
$$

Using (9), we see that in the market allocation

$$
E V_{Y}^{1}(p \theta-(1+r))=0 \text {. }
$$

$\left(16 c^{\prime}\right)$ and $\left(9^{\prime}\right)$ together imply that a necessary condition for the market to be a constrained Pareto optimum is that

$$
E B\left[\frac{d p}{d I_{2}}-\sum A^{i}\left(\hat{\beta}^{\prime} p \theta-\hat{\gamma}^{\prime}(1+r)\right)\right]=0 .
$$

Alternatively, using (18c), we can rewrite (23) as

$$
E B\left[\frac{-\frac{\partial C_{2}^{1}}{\partial Y}(p \theta-(1+r))}{\sum_{k}\left(\frac{\partial C_{2}^{k}}{\partial p}+\frac{\partial C_{2}^{k}}{\partial Y} \hat{\beta}^{k} \theta I_{2}\right)}\right]=0 .
$$

The central point of this paper is to show that (23) (or $23^{\prime}$ ) will be satisfied only under very restrictive conditions, far more restrictive than even those required for exchange efficiency.

If there is exchange efficiency, from (20)

$$
\hat{\beta}^{i} E A^{i} B p \theta=\hat{\beta}^{\prime} \frac{E V_{Y}^{i} p \theta}{E V_{Y}^{i}} E A^{\prime} B
$$


Using (9) and summing over $j$, we obtain

$$
E \sum \hat{\beta}^{i} A^{\prime} B p \theta=(1+r) \sum, E A^{i} B \hat{\beta}^{\prime} .
$$

Hence (23) becomes

$$
E B\left[\frac{d p}{d I_{2}}-\sum_{j} A^{i}\left(\hat{\beta}^{i}-\hat{\gamma}^{i}\right)(1+r)\right]=0 .
$$

The conditions for exchange efficiency clearly do not imply production efficiency. In particular, if $A^{i}=0$ (which, it will be recalled, is a sufficient condition for exchange efficiency), we still require that $E B d p / d I_{2}=0$. In the absence of income effects associated with redistribution, a necessary condition for the constrained Pareto optimality of the market is that

$$
\Omega \equiv E B \frac{d p}{d I_{2}}=0 .
$$

Using $\left(17^{\prime}\right)$ we can rewrite this as

$$
E \sum \lambda^{i} V^{\prime} T^{\prime} p \frac{d \ln p}{d I_{2}}=0 .
$$

Obviously, as we noted earlier, if the allocation investment had no effect on the relative price of the two goods $\left(d p / d I_{2} \equiv 0\right)$, then $\Omega=0$. But this is an uninteresting case, since it requires the two goods to be perfect substitutes.

Even if changing $I_{2}$ does not change relative profits in two states, i.e.

$$
\frac{d \ln p}{d I_{2}}=\text { constant, all } \theta,
$$

$\Omega$ will not, in general, be zero. We require, in addition, that

$$
E \sum \lambda^{j} V_{Y}^{\prime} p T^{j}=0
$$

i.e. that the (average) covariance between the value of "net trade" $\left(p T^{\prime}\right)$ and the marginal utility of income be zero.

Three sufficient conditions for (26) to hold are immediate:

(a) All individuals are identical; for then, as we noted earlier, $T^{j} \equiv 0$ and $B \equiv 0$.

(b) For all individuals $d V_{Y}^{\prime} / d \theta=0$, and the market has exchange efficiency. Exchange efficiency assures that $\lambda^{i} E V_{Y}^{i}=\lambda^{1} E V_{Y}^{1}$. Hence

$$
\sum_{i} E \lambda^{i} V_{Y}^{i} T^{j} \frac{d p}{d I_{2}}=\lambda^{1} V_{Y}^{1} E \sum_{i} T^{\prime} \frac{d p}{d I_{2}}=\lambda^{1} V_{Y}^{1} E \frac{d p}{d I_{2}} \sum T^{i}=0 .
$$

In the Appendix, we note that the condition $d V_{Y} / d \theta=0$ is far more restrictive even than the condition of income risk neutrality $\left(V_{Y Y}=0\right)$; even if all individuals had income risk neutrality, for $d V_{Y} / d \theta=0$, the individual's demand for the commodity must be independent of income.

(c) All individuals have Cobb-Douglas utility functions, for then, as we noted earlier, $p \theta$ is constant and $B \equiv 0$.

In this section we have established that the conditions for the constrained Pareto optimality of the market differ markedly from the conditions characterizing competitive market equilibria. In general, given the pattern of investment, the market will not 
allocate ownership claims on the different assets in a constrained Pareto optimal way; it will not have the property of exchange efficiency. And the market's allocation of investment among different industries will not be efficient. In both cases, the inefficiency arises from the fact that these allocation decisions affect the price distribution of the risky asset. As we noted in the introduction, it is as if we replaced one risky asset with another; the market ignores this effect, a "social planner" would not.

We have thus established a clear presumption against the (constrained) Pareto optimality of the market. It is not, however, obvious whether there are any sets of restrictions (say on the preferences of individuals) which ensure that nonetheless, the market is a constrained Pareto optimum. In the next two sections, we show that the only such conditions are precisely those that ensure that there is either no risk in the market or, even if there is risk, there is no trading on the stock market.

\section{NECESSARY CONDITIONS FOR OPTIMALITY WITH IDENTICAL PREFERENCES}

In the preceding section, we showed that the market allocations in general had neither the properties of exchange nor production efficiency. The conditions required for efficiency appear to be quite stringent.

Indeed, we were able to derive only three sufficient conditions ensuring efficiency, one of which was uninteresting (all individuals are identical), one of which made the risk market uninteresting (all individuals had Cobb-Douglas utility functions), and one of which was not directly related to the underlying specification of utility functions and technology for the economy (condition $b$ above). A natural question to ask is, are there weaker, sufficient conditions ensuring efficiency? Or have we detected here a generic source of market failure?

The objective of this and the next section is to show that there is, in fact, a strong presumption that market allocations will not be efficient. We do this by asking, for what classes of utility functions is it the case that, regardless of the specification of technologies and endowments, the market allocation will be efficient. ${ }^{8}$ Since our main concern is with the ability of the market to allocate investment efficiently, we focus on the case where the market has exchange efficiency; we assume all individuals have identical, homothetic indifference maps. This, as we noted earlier, means that redistribution of ownership claims has no effect on the price distribution (and it is this which ensures the exchange efficiency of the market.) Individuals still may differ in their attitudes towards risk, and these differences in risk aversion provide the basis of trade on the stock market.

We establish here that if all individuals have identical homothetic indifference maps, then the only conditions under which the market will generate a constrained Pareto optimal allocation of resources (for all specifications of technology and endowments) are

(i) all individuals have identical, constant relative risk aversion, so there is no trade on the stock market;

or

(ii) all individuals have unitary price elasticities of demand, so the value of output of each industry is fixed; there is essentially no risk and again, no trading on the stock market.

The assumption of the identical homothetic preferences implies that prices will be independent of the distribution of income. This greatly simplifies the analysis. The $j$-th individual's indirect utility function can be written

$$
\begin{gathered}
V^{i}=\phi^{i}\left(Y^{i} u(p)\right) \\
V_{Y}^{\prime}=\phi^{\prime \prime} u(p), V_{p}^{\prime}=\phi^{\prime \prime} Y^{i} u^{\prime} .
\end{gathered}
$$


Hence by Roy's identity

$$
C_{2}^{i}=-\frac{Y^{i} u^{\prime}}{u}
$$

Let $s^{i}=$ expenditure share of commodity 1 . Then

$$
s^{i}=\frac{C_{1}^{j}}{Y^{i}}=\frac{u^{\prime} p}{u}+1
$$

It is convenient to define

$$
\nu(p) \equiv \frac{1-s(p)}{s(p)}=-\frac{u^{\prime} p / u}{1+u^{\prime} p / u} .
$$

In equilibrium

$$
\nu(p)=\frac{1-s(p)}{s(p)}=\frac{p X_{2}}{X_{1}}=\frac{p \theta I_{2}}{(1+r) I_{1}} .
$$

Thus

$$
\frac{I_{2}}{p} \frac{d p}{d I_{2}}=\frac{\nu}{\nu^{\prime} p-\nu}\left(1+\frac{I_{2}}{I_{1}}\right)
$$

and

$$
-\frac{\theta}{p} \frac{d p}{d \theta}=-\frac{\nu}{\left(\nu^{\prime} p-\nu\right)} \equiv \frac{1}{\sigma}
$$

the elasticity of substitution. Thus

$$
\frac{\nu^{\prime} p}{\nu}=(1-\sigma)
$$

Substituting (30a) and (30b) into (26), and using (17'), we obtain

$$
-\Omega=\frac{\bar{I}}{I_{2} I_{1}} \sum E \lambda^{\prime} \phi^{\prime \prime} u(p) T^{\prime} \frac{p}{\sigma} \text {. }
$$

Let us consider the case of two groups, denoted by superscripts $a$ and $b$. Then $T^{a}=-T^{b}$. Given that the government has engaged in optimal lump sum transfers,

$$
E D u(p)=0
$$

where $D$ is the difference in (weighted) marginal utilities

$$
D \equiv \lambda^{a} \phi^{a^{\prime}}-\lambda^{b} \phi^{b^{\prime}}
$$

((31a) is just a special case of $16 a$.

Thus (21') can be rewritten as

$$
-\Omega \equiv \frac{\bar{I}}{I_{1} I_{2}} E \frac{D u(p) T^{a} p}{\sigma}=\frac{\bar{I}}{I_{1} I_{2}} E[D u(p)-E D u(p)]\left[\frac{T^{a} p}{\sigma}-E \frac{T^{a} p}{\sigma}\right] .
$$

From this it follows that a necessary condition for the optimality of the market for all distributions of $\theta$ is that

$$
\frac{T^{a} p}{\sigma}
$$

be independent of $\theta$, or that $D \equiv 0$. 
(a) Conditions for $D \equiv 0$. For $D \equiv 0$ for all values of the parameters requires that (letting $\lambda^{a} / \lambda^{b} \equiv \lambda$ )

$$
\frac{d \lambda}{\lambda} \equiv \rho^{a} \frac{d Y^{a}}{Y^{a}}-\rho^{b} \frac{d Y^{b}}{Y^{b}}
$$

where $\rho^{i}=\left(-V_{Y Y}^{\prime} Y^{\prime} / V_{Y}^{j}\right)=-\left(\phi^{i n} Y^{\prime} u / \phi^{\prime \prime}\right)=$ measure of relative risk aversion. Since

$$
\begin{gathered}
\frac{p \theta I_{2}}{(1+r) I_{1}}=\nu(p), \\
p \theta=k \nu(p),
\end{gathered}
$$

where $k=(1+r) I_{1} / I_{2}$.

Without loss of generality, we can write

$$
Y^{\prime}=M^{\prime}+N^{i} \nu(p)
$$

Thus

$$
d \ln \lambda \equiv \frac{\rho^{a}\left(d M^{a}+\nu(p) d N^{a}\right)}{M^{a}+\nu(p) N^{a}}-\rho^{b} \frac{d M^{b}+\nu(p) d N^{b}}{M^{b}+\nu(p) N^{b}}
$$

Since

$$
\frac{D}{\lambda^{b}} \equiv \lambda \phi^{a^{\prime}}\left[u(p)\left(M^{a}+N^{a} \nu\right)\right]-\phi^{h^{b}}\left[u(p)\left(M^{b}+N^{b} \nu\right)\right],
$$

$d D: d p=0$ implies

$$
\rho^{a}\left(\frac{u^{\prime} p}{u}+\frac{N^{a} \nu^{\prime} p}{M^{a}+N^{a} \nu}\right)=\rho^{b}\left(\frac{u^{\prime} p}{u}+\frac{N^{b} \nu^{\prime} p}{M^{b}+N^{b} \nu}\right)
$$

or

$$
\rho^{a}\left(-(1-s)+\frac{N^{a}}{Y^{a}} \frac{(1-s)}{s}(1-\sigma)\right)=\rho^{b}\left(-(1-s)+\frac{N^{b}}{Y^{b}} \frac{(1-s)}{s}(1-\sigma)\right) .
$$

Consider now a perturbation in the parameters of the problem (keeping $\lambda$ constant). Clearly, from (33')

$$
\rho^{a} \frac{d M^{a}+\nu d N^{a}}{Y^{a}}=\rho^{b} \frac{d M^{b}+\nu d N^{b}}{Y^{b}} .
$$

Dividing (37) by ( $\left.36^{\prime}\right)$, we obtain

$$
\frac{d M^{a}+\nu d N^{a}}{(1-s)\left[N^{a} \frac{(1-\sigma)}{s}-Y^{a}\right]}=\frac{d M^{b}+\nu d N^{b}}{(1-s)\left[N^{b} \frac{(1-\sigma)}{s}-Y^{b}\right]}
$$

or

$$
d M^{a}+\nu d N^{a}=\frac{N^{a} \frac{(1-\sigma)}{s}-Y^{a}}{N^{b} \frac{(1-\sigma)}{s}-Y^{b}}\left(d M^{b}+\nu N^{b}\right) .
$$

The LHS of $\left(38^{\prime}\right)$ is linear in $\nu$. Hence the RHS must be linear in $\nu$. Hence

$$
N^{a}(s-\sigma)-s M^{a}=\kappa\left[N^{b}(s-\sigma)-s M^{b}\right]
$$


or

$$
\frac{(s-\sigma)}{s}\left(N^{a}-\kappa N^{b}\right)=M^{a}-\kappa M^{b} .
$$

Thus, either $(s-\sigma) / s$ is constant or $N^{a}-\kappa N^{b}=M^{a}-\kappa M^{b}=0$ :

$$
\text { (i) } \frac{s-\sigma}{s}=\text { constant; }
$$

there is a special family of utility functions for which this is true; it is found by solving the differential equation

$$
\sigma=1-\frac{\nu^{\prime} p}{\nu}=c \cdot \frac{1}{1+\nu}=c \cdot s
$$

for some value of $c$; and then solving the differential equation

where

$$
\frac{d \ln u}{d \ln p}=-\frac{\nu^{*}(p)}{1+\nu^{*}(p)}
$$

$$
\nu^{*}(p) \text { is the solution to }(41) \text {. }
$$

Within the class of constant elasticity functions, the only utility function satisfying $(40 a)$ is (trivially) the unitary elasticity of substitutions, i.e. $p \theta$ is constant, so there is no risk.

In the analysis so far, we have assumed that all of the variability in output occurred in sector 2. By identical arguments, if all the variability in output occurs in sector 1 , we require that

$$
\frac{\sigma}{1-s}=\text { constant }
$$

(40a) and (40b) together imply that $\sigma$ is constant, and our previous argument thus establishes that if $D=0$ for all $p$ for all feasible parameters of the problem, individuals must have unitary price elasticities.

$$
\text { (ii) } M^{a}=\kappa M^{b}, \quad N^{a}=\kappa N^{b} .
$$

The two individuals have identical portfolio allocations, i.e. they must have identical (constant) relative risk aversions, or there is no risk $(\sigma=1)$.

(b) Condition for $T^{a} p / \sigma$ to be constant. Similarly, $T^{a} p / \sigma$ will be constant only under special conditions. Recalling (22) and (28)

$$
\begin{aligned}
p T^{a} & =\beta^{a} p \theta I_{2}-(1-s(p)) Y^{a} \\
& =\beta^{a} p \theta I_{2}\left(1-\frac{\nu}{1+\nu}\right)-\frac{\nu \gamma^{a}}{1+\nu} I_{1}(1+r) \\
& =\left[\beta^{a}-\gamma^{a}\right] I_{1}(1+r) \frac{\nu}{1+\nu} .
\end{aligned}
$$

$p T^{a} / \sigma$ must be constant, for all values of $p, \beta^{a}$, and $\gamma^{a}$. Hence either $\beta^{a} \equiv \gamma^{a}$ (all individuals are identical) or

$$
\frac{\nu}{(1+\nu) \sigma}=\frac{1-s}{\sigma} \equiv \text { constant, }
$$

precisely the same condition $((40 \mathrm{~b}))$ derived earlier for $D \equiv 0$. 
Hence, we have shown that if all individuals have identical homothetic indifference maps, then, if for all technologies, the market allocation is to be a constrained Pareto optimum, either all individuals must be identical or $p \theta$ must be constant. In both of these cases, the stock market is not performing any function in sharing or spreading risks, in the first because all individuals are identical, in the second because $p \theta$ is constant, and hence there is no risk in this economy.

\section{DIFFERENCES IN TASTES}

In the previous section, we showed that if all individuals have identical indifference maps, but differ only in their attitudes towards risks, then the only case in which the market is a constrained Pareto optimum (for all technologies) is that where all individuals have unitary price elasticity.

In this section, we establish a slightly more general result: even if individuals have different indifference maps, if all individuals have unitary price elasticities, the market is a constrained Pareto optimum but only because there is no market risk- $p \theta$ is constant.

On the other hand, we also show that this result is not robust. A slight change in the market structure-the introduction of a futures market on the price $p$ - has two important consequences:

(a) There is now aggregate market risk (even with all individuals having unitary' price elasticity demand curves); and

(b) The market is not a constrained Pareto optimum, except in the singular case, discussed in the preceding section, where all individuals have identical indifference curves and unitary price elasticities.

To obtain simple results we assume two groups. The $j$-th individual spends $s^{\prime}$ of his income on commodity 1 , with $0<s^{\prime}<1$.

Thus, we can write his utility function as some concave transform of a Cobb-Douglas function

$$
U^{\prime}=\Phi^{i}\left(\left(C_{1}^{\prime}\right)^{s^{\prime}}\left(C_{2}^{\prime}\right)^{1-s^{\prime}}\right), \quad \Phi^{i^{\prime}}>0, \quad \Phi^{i^{\prime \prime}}<0,
$$

generating an indirect utility function of the form

$$
V^{\prime}=\phi^{\prime}\left(\alpha^{i} p^{s^{\prime-1}} Y^{i}\right) \text {, where } \alpha^{\prime}=\left(s^{\prime}\right)^{s^{\prime}}\left(1-s^{\prime}\right)^{1-s^{\prime}} \text {. }
$$

(the special case of $(27)$ where $u(p)=p^{s-1}$ ).

Since one of our objectives in this section is to see the effect on aggregate risk of introducing an additional market-a gamble on the price of $X_{2}$ next period (this is called a futures market), we let $\delta^{\prime}$ be the purchases (sales) of the $j$-th group on the futures market and $q$ be the present price. Then

$$
Y^{\prime}=\beta^{i} p \theta I_{2}+(1+r)\left[I^{j}-\delta^{\prime} q-\beta^{j} I_{2}\right]+\delta^{\prime} p
$$

If $\delta^{i}$ is chosen optimally,

$$
E V_{Y}^{i} \frac{p}{q}=E V_{Y}^{t}(1+r)
$$

or

$$
\frac{E V_{Y p / q}^{i}}{E V_{Y}^{j}}=1+r
$$

Again, the weighted average returns must be the same as those on a safe asset.

Now in equilibrium demand for good 1 must equal supply,

$$
I_{1}(1+r)=s^{a} Y^{a}+s^{b} Y^{b}
$$

and demand for "futures" must equal supply, i.e.

$$
\delta^{a}=-\delta^{b}
$$


Thus using (45)

$$
I_{1}(1+r)=\left(s^{a} \beta^{a}+s^{b} \beta^{b}\right) p \theta I_{2}+\left(s^{a}-s^{b}\right) \delta^{a} p+k I_{1}(1+r)
$$

where

$$
k=\left(s^{a} \gamma^{a}+s^{b} \gamma^{b}\right)
$$

(48) has one important implication: $p \theta$ is a linear function of $p$ :

$$
p \theta=\frac{I_{1}(1+r)(1-k)-\left(s^{a}-s^{b}\right) \delta^{a} p}{I_{2}\left(s^{a} \beta^{a}+s^{b} \beta^{b}\right)}=z_{1}+z_{2} p .
$$

Substituting (49) back into (45), we see that $Y^{j}$ is linear in $p$ :

where

$$
Y^{\prime}=Q^{j}+R^{\prime} p
$$

$$
\begin{gathered}
Q^{\prime}=(1+r)\left[I^{j}-\delta^{\prime} q-\beta^{i} I_{2}\right]+\frac{\left\{\beta^{i} I_{1}(1+r)(1-k)\right\}}{s^{a} \beta^{a}+s^{b} \beta^{b}} \\
R^{\prime}=\delta^{\prime}-\frac{\beta^{\prime}\left(s^{a}-s^{b}\right) \delta^{a}}{s^{a} \beta^{a}+s^{b} \beta^{b}} .
\end{gathered}
$$

This fact will prove to be important in our later analysis. (49) in turn has an important implication: in the absence of a futures market $(\delta \equiv 0)$, if all individuals have unitary price elasticity, but different indifference maps (shares of expenditure on the two commodities), the aggregate demand curve will also have unitary price elasticity; there will be no market risk, and the market allocation will therefore be a constrained Pareto optimum. As soon as we introduce a futures market, however, the aggregate demand curve will not have unitary price elasticity and the market allocation will not be a constrained Pareto optimum.

To establish this we will need to make use of the first order conditions for a constrained Pareto optimum and the special properties of our demand functions. In particular, we note that (using (48))

$$
\begin{aligned}
& \frac{d \ln p}{d I_{2}}=-\frac{\tilde{s} p \theta+(1-k)(1+r)}{I_{1}(1-k)(1+r)} \equiv-\frac{m_{1} p \theta+1}{I_{1}} \\
& \frac{d \ln p}{d \gamma^{a}}=-\frac{\left(s^{a}-s^{b}\right)}{1-k} \equiv m_{2} \\
& \frac{d \ln p}{d \beta^{a}}=-\frac{\left(s^{a}-s^{b}\right) p \theta I_{2}}{I_{1}(1-k)(1+r)} \\
& \frac{d \ln p}{d \delta^{a}}=-\frac{\left(s^{a}-s^{b}\right) p}{I_{1}(1-k)(1+r)}
\end{aligned}
$$

where $\bar{s}=s^{a} \beta^{a}+s^{b} \beta^{b}$.

The first order condition for exchange efficiency can now be written as

$$
\begin{array}{r}
E D\left(1-\frac{p T^{a}\left(s^{a}-s^{b}\right)}{I_{1}(1-k)(1+r)}\right)=0 \\
E D\left(1-\frac{p T^{a}\left(s^{a}-s^{b}\right)}{I_{1}(1-k)(1+r)}\right) p \theta=0 \\
E D\left(1-\frac{p T^{a}\left(s^{a}-s^{b}\right)}{I_{1}(1-k)(1+r)}\right) p=0
\end{array}
$$


where now

$$
T^{\prime} \equiv \beta^{\prime} \theta I_{2}+\delta^{\prime}-c_{2}^{\prime}
$$

Now observe that $p T^{a}$ is linear in $p$ :

$$
\begin{aligned}
p T^{a} & =p \beta^{a} \theta I_{2}-\left(1-s^{a}\right) Y^{a}+p \delta^{a} \\
& =s^{a}\left(\beta^{a} p \theta I_{2}+\delta^{a} p\right)-\left(1-s^{a}\right) \gamma^{a} I_{1}(1+r) \\
& =s^{a} \beta^{a} I_{2} z_{1}-\left(1-s^{a}\right) \gamma^{a} I_{1}(1+r)+s^{a} p\left(z_{2} \beta^{a} I_{2}+\delta^{a}\right) \\
& \equiv z_{3}^{\prime}+\frac{s^{a} s^{b} \delta^{a}}{\bar{s}} p .
\end{aligned}
$$

Hence (53) can be rewritten as

$$
E D\left(z_{3}+z_{4} p\right)=0, \quad E D\left(z_{3}+z_{4} p\right) p=0
$$

where

$$
z_{3}=-\frac{z_{3}^{\prime}\left(s^{a}-s^{b}\right)}{I_{1}(1-k)(1+r)}+1, \quad z_{4}=-\frac{\left(s^{a}-s^{b}\right) s^{a} s^{b} \delta^{a}}{I_{1}(1-k)(1+r) \vec{s}} .
$$

The first order condition for production efficiency can be written

$$
E \sum \lambda^{\prime} V_{Y}^{\prime}\left(\beta^{\prime} p \theta-\gamma^{\prime}(1+r)\right)+E D p T^{a} \frac{d \ln p}{d I_{2}}=0 .
$$

In the market equilibrium

$$
E V_{Y}^{i} p \theta=E V_{Y}^{i}(1+r)
$$

so for the market equilibrium to be a constrained Pareto optimum

$$
(1+r) E D\left(\beta^{a}-\gamma^{a}\right)+E D p T^{a} \frac{d \ln p}{d I_{2}}=0 .
$$

The portfolio equilibrium condition implies

$$
\begin{aligned}
& \lambda^{a} E V_{Y}^{a} p \theta=\lambda^{a} E V_{Y}^{a}(1+r) \\
& \lambda^{b} E V_{Y}^{b} p \theta=\lambda^{b} E V_{Y}^{b}(1+r) .
\end{aligned}
$$

Subtracting

$$
E D p \theta=(1+r) E D .
$$

Substituting (63) and (52a) into (60), we obtain (after some simplification)

$$
E D=0 \text {. }
$$

But from (49) and (63),

$$
E D p \theta=z_{1} E D+z_{2} E D p=(1+r) E D .
$$

Hence either

$$
z_{2}=0
$$

or

$$
E D p=0 .
$$

If $z_{2}=0, p \theta$ is constant: there is no risk. 
Using (64) and (67), we can now rewrite the condition for production efficiency as

$$
E D p T^{a} \frac{d \ln p}{d I_{2}}=-\frac{s^{a} s^{b} \delta^{a}}{I_{1}(1-k)(1+r)} E D p^{2} \theta=0 .
$$

This requires ${ }^{9}$ either

$$
p \theta \text { be constant }
$$

or

$$
D \equiv 0
$$

We now see what each of these entails:

(a) $p \theta=$ constant implies $z_{2}=0$, which implies either

$$
s^{a}=s^{b}
$$

the individuals have the same indifference map (the case studied in the previous section); or

$$
\delta^{a}=0
$$

For $\delta^{a}=0$

$$
\frac{V_{Y P}^{a}}{V_{Y}}=\frac{V_{Y p}^{b}}{V_{Y}}
$$

i.e. since there is no risky income,

$$
\begin{gathered}
V_{p}^{a}=-C_{2}^{a} V_{Y}^{a} \\
-\frac{p V_{p Y}^{a}}{V_{Y}}=s\left(1-s^{a}\right)\left(1-\rho^{a}\right) .
\end{gathered}
$$

Hence, we require

$$
\left(1-s^{a}\right)\left(1-\rho^{a}\right)=\left(1-s^{b}\right)\left(1-\rho^{b}\right)
$$

(b) $D \equiv 0$.

For simplicity, we limit ourselves to the case of constant relative risk aversion; we can then write

$$
\begin{aligned}
V^{\prime} & =\phi^{\prime}\left(Y^{i} p^{s^{\prime-1}}\right) \\
& =\frac{\left[Y^{\prime} p^{s^{\prime-1}}\right]^{1-\rho^{\prime}}}{1-\rho^{\prime}} \\
& =\frac{\left[\left(Q^{i}+R^{\prime} p\right) p^{s^{\prime-1}}\right]^{1-\rho^{\prime}}}{1-s^{\prime}}
\end{aligned}
$$

(using (50)).

Thus for $D \equiv 0$,

$$
\lambda^{a} p^{\left(s^{a}-1\right)\left(1-\rho^{a}\right)}\left(Q^{a}+R^{a} p\right)^{-p^{a}}=\lambda^{b} p^{\left(s^{b}-1\right)\left(1-p^{b}\right)}\left(Q^{b}+R^{b} p\right)^{-\rho^{b}}
$$

for all $p$, or differentiating logarithmically,

$$
-\left(1-s^{a}\right)\left(1-\rho^{a}\right)-\frac{\rho^{a} R^{a} p}{Q^{a}+R^{a} p}=-\left(1-s^{b}\right)\left(1-\rho^{b}\right)-\frac{\rho^{b} R^{b} p}{Q^{b}+R^{b} p} .
$$

Multiply through by $Q^{b}+R^{b} p$ and $Q^{a}+R^{a} p$, and differentiating twice with respect to $p$, we obtain

$$
-\left(1-s^{a}\right)\left(1-\rho^{a}\right) R^{a} R^{b}-\rho^{a} R^{a} R^{b}=\left(1-s^{b}\right)\left(1-\rho^{b}\right) R^{a} R^{b}-\rho^{b} R^{a} R^{b}
$$


or

$$
R^{a} R^{b} s^{a}\left(1-\rho^{a}\right)=R^{a} R^{b} s^{b}\left(1-\rho^{b}\right)
$$

which implies either

$$
R^{a} \text { or } R^{b}=0
$$

1.e.

$$
\delta^{a}\left[1-\frac{\beta^{a}\left(s^{a}-s^{b}\right)}{\bar{s}}\right]=\frac{s_{b}}{\bar{s}} \delta^{a} .
$$

But this requires $\delta^{a}=0$; or

$$
s^{a}\left(1-\rho^{a}\right)=s^{b}\left(1-\rho^{b}\right),
$$

which after some manipulation, can again be shown to imply $\delta^{a}=0$.

In either case, there is no risk in the market.

In short, if all individuals have unitary price elasticities, but different preferences, except in a singular case where there is no variability in profits ( $p^{*}$ is constant) the market equilibrium will not be a constrained Pareto optimum for all specification of the technology.

We established that if all individuals have unitary price elasticities, if there were no futures market, the market would be a constrained optimum and there would be no risk. The introduction of a futures market will increase the variability of all individual's income (using the random commodity as numeraire); we have not, however, determined conditions under which the opening of the futures market constitutes a Pareto improvement or, alternatively, makes all individuals worse off.

\section{CONCLUDING COMMENTS}

This paper is a contribution to the growing literature which has developed from Karl Borch's seminal work; this research has cast doubt on the efficiency of the economy in allocating resources in the presence of an incomplete set of risk markets.

This recent literature has questioned:

(a) the appropriateness of the criterion of stock market value maximization

(b) the persuasiveness of the assumption that the value of firms will increase in proportion to their scale and

(c) the generality of the result that the market will be a constrained Pareto optimum

(d) the optimality of the set of markets which are in existence.

This paper has considered a situation in which the technology is such that all shareholders would wish the firm to maximize the stock market value and in which the hypothesis that the value of the firm increases in proportion to its scale is plausible. Nonetheless, we have shown that there is a strong presumption that the market is not a constrained Pareto optimum; it is not just that "examples" can be found in which the market equilibrium is a Pareto optimum. Rather, the basic marginal conditions describing the market equilibrium and the optimum are different. We have shown here that the set of circumstances in which the two conditions coincide are very restrictive. In general, competitive markets lack both exchange and production efficiency. Exchange efficiency obtains if all individuals have identical indifference maps, but even this restriction is not enough to ensure efficiency of the allocation of investment. For that, we require all individuals to be identical or have unitary price elasticities. Both of these cases imply the stock market performs no role in risk sharing.

With incomplete markets, prices are performing two functions: not only do they have their conventional role in allocating resources, they perform a critical function in 
sharing and transferring risk (insurance). When, for instance, price and quantity vary inversely, the risk of production is transferred from producers to consumers by the price system. Evidently, when too much is asked of the price system, it does not perform well, or at least, as well as it would with selective government intervention.

(It is important to realize that the kind of argument we employ here is markedly different from the naive argument that the market does not allocate resources efficiently because producers will not undertake sufficient risk. If there are incomplete risk markets, the risks which are borne by producers (which in a complete set of markets they might be able to insure against) have a real social cost; that is, the fact that the risks might, under some other institutional arrangement, be spread does not alter the fact that under the institutional arrangement under consideration, producers do in fact bear a risk which affects their expected utility. The conventional discussions of the issue seem to make the error of comparing the allocation of resources with one market structure with the allocation which would have emerged with another market structure, a comparison which we have argued is unfair, and probably irrelevant. In our analysis, we have explicitly taken into account the costs of risk as they are actually borne in the second-best situation under consideration.)

The inefficiencies with which we have been concerned here (and in Stiglitz 1972a, $1975 b$ ) are marginal inefficiencies; in addition, it is important to remember as Drèze (1974), Hart (1975), and Stiglitz (1972a) have emphasized that there may be, in such situations, structural inefficiencies: given the set of production decisions made by other firms, each firm is making the correct decisions; yet, there exists another set of (mutually consistent) actions which leads to a Pareto improvement. There are, in other words, multiple Nash equilibrium, some of which Pareto dominate others.

There are two marked limitations of our analysis which should be noted. First, we have not provided a quantitative estimate of the magnitude of the distortions (or the welfare losses to which they give rise); indeed, we have not even obtained a clear qualitative analysis of the direction of the bias. (Our analysis does shed some light on the kinds of parameters which are likely to be relevant, e.g. the magnitude of differences in tastes and risk aversion.) Although, in principle, a set of taxes-subsidies could be devised which would enable the economy to attain a constrained Pareto optimum, the magnitude of the taxes and subsidies may vary markedly from industry to industry and depend on parameters (probability distributions of returns) which are difficult to estimate with any degree of accuracy. Thus the implementation of such tax-subsidy schemes may be extraordinarily difficult; our analysis may perhaps be better viewed as casting doubt on the validity of the claims that the market provides an efficient allocation of resources rather than as a basis of a prescriptive theory of government intervention.

Secondly, we have not provided an explanation of the limitations on the set of markets which function. The kind of analysis which we have employed here, analyzing the performance of the economy with a given set of markets, and then evaluating whether there is an optimal set of markets in operation, is clearly a second best approach to a second best problem: it would be desirable to analyze both problems simultaneously. This is the next task on our research agenda. ${ }^{10}$

\section{APPENDIX}

Derivation of Conditions for Marginal Utility of Income to be Independent of State

The condition $d V_{Y} / d \theta=0$ is a very stringent condition. To see what it implies observe that

$$
\frac{d V_{Y}^{\prime}}{d p}=V_{Y_{p}}^{\prime}+V_{Y Y}^{\prime}\left(1-\alpha^{\prime}\right) \theta I^{i}
$$


But, using Roy's formula,

$$
V_{p}^{i}=-C_{2}^{i} V_{Y}^{i}
$$

Differentiating, we obtain

$$
V_{p Y}^{\prime}=-C_{2}^{i} V_{Y Y}-V_{Y}^{\prime} \frac{d C_{2}^{j}}{d Y} .
$$

Thus, (using A.1) and (22)

$$
\frac{d V_{Y}^{\prime}}{d p}=-V_{Y}^{\prime}\left[\frac{d C_{2}^{i}}{d Y}+\left(\frac{-V_{Y Y}^{i}}{V_{Y}^{\prime}}\right) T^{\prime}\right] .
$$

Let

$$
\begin{gathered}
\rho^{i}=\frac{-V_{Y Y}^{i} Y^{j}}{V_{Y}^{\prime}}=\text { measure of relative risk aversion } \\
\eta^{\prime}=\frac{d \ln C_{2}^{j}}{d \ln Y^{\prime}}=\text { measure of elasticity of demand }
\end{gathered}
$$

and

$$
-\frac{1}{\sigma} \equiv \frac{\theta}{p} \frac{d p}{d \theta}=\frac{d \ln p}{d \ln X_{2} / X_{1}}
$$

( $\sigma$ is the "aggregate" elasticity of substitution).

Then (using (22), (A.3)-(A.6))

$$
\begin{aligned}
\frac{d V_{Y}^{\prime}}{d \theta} & =\frac{d V_{Y}^{i}}{d p} \frac{d p}{d \theta}+V_{Y Y}^{\prime}\left(1-\alpha^{\prime}\right) p I^{\prime} \\
& =\frac{V_{Y}^{\prime} C_{2}^{j} p}{\theta Y^{\prime}}\left\{\left(\eta^{i}+\rho^{\prime} \frac{T^{j}}{C_{2}^{\prime}}\right) \frac{1}{\sigma}-\rho^{\prime}\left(1+\frac{T^{\prime}}{C_{2}^{\prime}}\right)\right\}=0
\end{aligned}
$$

if and only if (assuming $\sigma \neq 1$ )

$$
\frac{T^{\prime}}{C_{2}^{i}} \equiv-\frac{\rho^{\prime} \sigma-\eta^{j}}{\rho^{\prime}(\sigma-1)} \text { for all } \theta .
$$

Clearly (A.8) is very restrictive. For instance, if there is constant income elasticity, constant relative risk aversion and constant aggregate elasticity of substitution, then for (A.8) to hold identically for all $\theta$ requires $C_{2}^{\prime}$ to be proportional to $\theta$ for all $j$, i.e. the proportion of the total output of the second commodity consumed by each individual is the same in all states of nature.

\section{NOTATION}

$\begin{array}{ll}p & \text { price of commodity } 2 \text { (relative to commodity } 1 \text { ) } \\ Y^{i} & \text { income of } j \text {-th individual (in commodity } 1 \text { numeraire) } \\ X_{i} & \text { output of commodity } i \\ I_{i} & \text { investment in industry } i \\ I^{j} & j \text {-th individual's initial wealth } \\ I & \text { aggregate investment } \\ \alpha^{i} & \text { proportion of } j \text {-th individual's initial wealth invested in industry } 1 \\ \beta^{j} & \text { proportion of industry } 2 \text { owned by } j \text {-th individual } \\ \gamma^{\prime} & \text { proportion of industry } 1 \text { owned by } j \text {-th individual }\end{array}$




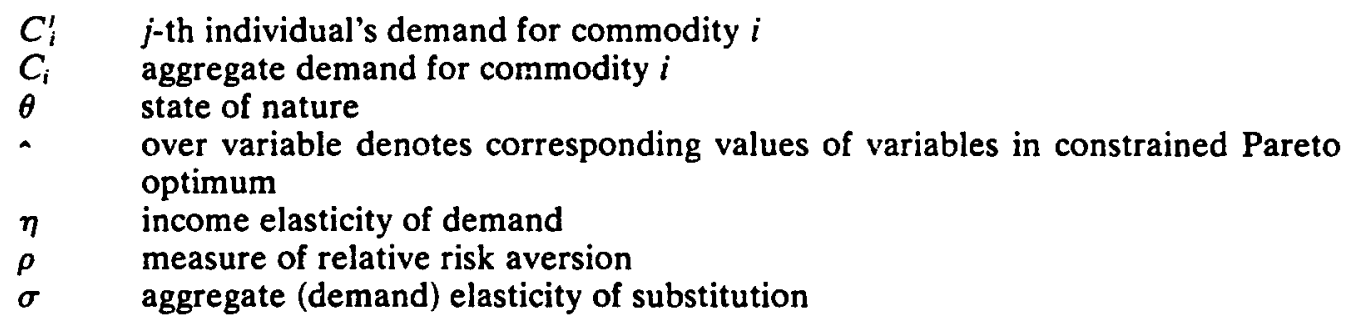

First version received September 1979, final version accepted September 1981 (Eds.).

Paper prepared for a conference in Bergen, Norway, April 26-27, 1979, in honour of Karl Borch. This is a revised and simplified version of Part I of "The Inefficiency of the Competitive Stock Market and its Implications for the Depletion of Natural Resources" (Stanford, mimeo, 1975). I am indebted to J. Mirrlees, Sandy Grossman, Barry Nalebuff, Robin Lindsey, Oliver Hart, Partha Dasgupta, Vijay Krishna and the referee for helpful suggestions. Financial support from the National Science Foundation is gratefully acknowledged. This paper was completed while 1 was a visitor at the Department of Economics and the Center for Social Sciences at Columbia University, to whom I am indebted for their hospitality.

\section{NOTES}

1. Even this minimal test of market efficiency may be misleading: the reasons which resulted, in the first place, in the absence of the risk markets may make the proposed taxes and subsidies either not feasible or extremely costly. This qualification does not, however, seem to have much force in the particular context examined here. Without loss of generality, we can allow them also to be state dependent, but if so, to be linearly dependent on the outputs of the different firms in the econom!

2. Although we do not do so here, it is easy to establish (using, for instance, the analysis of GrossmanStiglitz, 1980) that if all firms believe that they have no effect on the probability distribution of prices, all firms face technological stochastic homotheticity (the ratio of outputs in any two states is independent of scale), and the only choice facing firms is that of scale, then all shareholders will wish the firm to maximize the market value.

3. We ignore the well known problems associated with bankruptci. See Stiglitz (1972b) and $(1975 b)$ and Grossman-Stiglitz (1980).

4. The issue of futures markets does not arise in Diamond's analysis, since there is only one commodity.

5. Hart (1975) provides an example of multiple equilibria in which in one of the equilibria all individuals are better of than in another. This is an example of what I have called elsewhere a structural inefficiency (Stiglitz (1972a 1). One of the Nash equilibria is Pareto optimal, but there is no way of ensuring that this is the one which will occur.

On the other hand, the inefficiency we discuss here (and which is the focus of concern in Stiglitz (1975a)) is a marginal inefficiency; at the margin, the private market makes incorrect investment decisions. This would be the case even were there a unique equilibrium. Hart makes a similar distinction in his (1975) paper, where he develops a notion of optimality, called strong optimality, which captures the idea of marginal inefficiency. In particular, an equilibrium may be strongly suboptimal even if it is unique.

6. More precisely, (i) and (ii) are sufficient conditions for the first-order conditions (16a) and (16b) to coincide with (9), the market equilibrium conditions. It is still possible that the economy not have exchange efficiency, if there are multiple solutions to (16), and the market allocation corresponds to either a local (but not global) maximum or to a minimum.

7. Since there is no risk, $V_{Y}^{\prime}$ is constant, and $\lambda^{\prime} V_{Y}^{i}=\lambda^{1} V_{Y^{\prime}}^{1}$ so

$$
B=\lambda^{\prime} V^{\prime} Y T^{\prime}=0 \text {. }
$$

The proof that $p \theta$ is constant is straightforward but tedious, and is postponed until Section 4 .

8. It should be clear from our arguments that we could alternatively have provided a generic inefficiency theorem, of the form "except under the conditions noted below, for an arbitrarily specified set of utility functions, the set of endowments and probability distributions of $\theta$ for which the economy has a constrained Pareto optimum is of measure zero".

9. If $p \theta$ is not constant (i.e. $z_{2} \neq 0$ ), and $D \neq 0$, then we can find probability distributions for which, even though in the competitive allocation, $E D=0, E D p=0, E D p^{2} \theta=z_{2} E D p^{2} \neq 0$. To see this most simply, note that the competitive allocation requires

$$
\begin{gathered}
E V_{Y}^{i}(p \theta-(1+r))=0, \quad i=a, b \\
\frac{E V_{Y p}^{a}}{E V_{Y}^{a}}=\frac{E V_{Y p}^{b}}{E V_{Y}^{b}}=q .
\end{gathered}
$$


164 ) and (67) together imply that (72) is satisfied. Let us fix $\left[I_{1}, I_{2}, \gamma^{a}, \beta^{a}, \delta^{a}\right]$, and hence $Y^{a}$ and $p$ for each $\theta$. These values are consistent with competitive equilibrium if (71) and (72) are satisfied. Let us now look at the subset of these for which

$$
\frac{E V_{Y}^{a}}{E V_{Y}^{b}}=\frac{\lambda^{b}}{\lambda^{a}}, \text { a constant. }
$$

(This is, of course, equivalent to (64)). (64), (67), and (71) define four linear equations in the probabilities of the different states. The restriction

$$
E D p^{2} \theta=0
$$

is thus an additional linear restriction, which will be satisfied on a set of measure zero unless $D p \cdot p \theta$ is a linear function of $D p, D$, and $V_{Y}^{a}(p \theta-(1+r))$ and $V_{Y}^{h}(p \theta-(1+r))$, which it will be only if $p \theta$ is a constant or $D=0$.

10. In research currently under way, we have gone some way towards this objective; in situations where the reason for the imperfect risk markets is moral hazard (related to the unobservability of individuals" actions), the market equilibrium is not, in general, a constrained Pareto optimum. (Arnott and Stiglitz, 1980.)

\section{REFERENCES}

ARNOTT, R. and STIGLITZ, J. E. (1980), "Moral Hazard and the Inefficiency of Competitive Equilibrium" (mimeo, paper prepared for Conference at Bell Laboratories).

ARROW, K. J. (1964), "The Role of Securities in the Optimal Allocation of Risk-Bearing", Revien of Economic Studies, 31, 91-96.

BORCH, K. (1962), "Equilibrium in a Reinsurance Market", Econometrica, 30, 424-444.

BORCH, K. (1960), "The Safety Loading of Reinsurance Premiums", Skandinavisk Aktuarietidskrift, $163-184$.

BORCH, K. (1968), "General Equilibrium in the Economics of Uncertainty", in Borch, K. and Mossin, J. (eds.) Risk and Uncertainty (London: Macmillan) 47-58.

BORCH, K. (1968). The Economics of Uncertainty (Princeton University Press).

DIAMOND, P. (1967), "The Role of a Stock Market in a General Equilibrium Model with Technological Uncertainty", American Economic Review, 57, 759-776.

DRĖZE, J. H. (1974), "Investment under Private Ownership: Optimality, Equilibrium and Stability", in Drèze, J. H. (ed.) Allocation under Uncertainty: Equilibrium and Optimality (Macmillan) Chapter 9.

GROSSMAN, S. J. and STIGLITZ, J. E. (1977), "On Value Maximization and Alternative Objectives of the Firm", Journal of Finance, 32 (2), 389-402.

GROSSMAN, S. J. and STIGLITZ, J. E. (1980), "Stockholder Unanimity in Making Production and Financial Decisions", Quarterly Journal of Economics, 525-542.

HART, O. (1975), "On the Optimality of Equilibrium when the Market Structure is Incomplete", Journal of Economic Theory, 11, 418-443.

JE.JSEN, M. C. and LONG, J. B. Jr. (1972), "Corporate Investment under Incertainty and Pareto Optimality in the Capital Markets", Bell Journal of Economics and Management Science, 3 (1), 151-174.

NEWBERY, D. and STIGLITZ, J. E. (1979), "Pareto Inferior Trade" (mimeo).

STIGLITZ, J. E. (1972a), "On the Optimality of the Stock Market Allocation of Investment", Quarterly Journal of Economics, 25-60.

STIGLITZ, J. E. (1972b), "Some Aspects of the Pure Theory of Corporate Finance Bankruptcies and Take-Overs", Bell Joumal of Economics and Management Science, 2, 458-482.

STIGLITZ, J. E. (1975a), "The Efficiency of Market Prices in Long Run Allocations in the Oil Industry", in Brannon, G. (ed.) Studies in Energy Tax Policy (Cambridge, Mass: Ballinger Publishing Co.) 55-99.

STIGLITZ, J. E. (1975b), "Reply to Mr. Singleton on "Some Aspects of the Pure Theory of Corporate Finance: Bankruptcies and Take-Overs' ", Bell Journal of Economics and Management Science, 16 (2), 711-714. 
(C) 1982 Review of Economic Studies Ltd 